\title{
Dynamics in Poly(n-alkyl methacrylates): A neutron scattering, calorimetric and dielectric study
}

A. Arbe ${ }^{1}$, A.-C. Genix ${ }^{2}$, S. Arrese-Igor ${ }^{1}$, J. Colmenero ${ }^{1,3}$, D. Richter ${ }^{4}$

1 Centro de Física de Materiales (CSIC-UPV/EHU), Apartado 1072, 20080 San Sebastián, Spain

2 Laboratoire des Colloïdes, Verres et Nanomatériaux,

UMR 5587 CNRS, Université Montpellier II, 34095 Montpellier, France

3 Departamento de Física de Materiales (UPV/EHU), Materials Physics Center (MPC), Apartado 1072,

20080 San Sebastián, Spain and Donostia International Physics Center,

Paseo Manuel de Lardizabal 4, 20018 San Sebastián, Spain and

${ }^{4}$ Institut für Festkörperforschung, Forschungszentrum Jülich GmbH, D-52425 Jülich, Germany

Combining neutron diffraction, neutron spin echo, differential scanning calorimetry and dielectric spectroscopy we have investigated the structure and dynamics of poly(n-butyl methacrylate) (PBMA) and poly(n-hexyl methacrylate) (PHMA). Signatures of the occurrence of a glass-transition associated to the freezing of the inter-molecular correlations within alkyl nanodomains are present in the structural data. Exploiting isotopic labeling, neutron scattering has revealed collective dynamics at the main-chain and side-group levels for both polymers and the self-motions of hydrogen atoms in the side-groups of PHMA, adding valuable microscopic information to comprehensive relaxation maps and putting the relaxation results into a perspective. Moreover, we find exotic dynamical behavior for the side-groups, char- 
acterized by extremely stretched (nearly logarithmic-like) decays of the correlation functions. For PHMA, a complete dynamical decoupling of side-group dynamics from the main-chain motions is found. The side-groups of this polymer show an extremely 'strong' temperature dependence of the structural relaxation time and much faster characteristic times for self than collective motions. The analogies found between the self-motions of the side-group H-atoms in PHMA and the $\gamma$-relaxation process in semicrystalline polyethylene $(\mathrm{PE})$ strengthen the picture of confined PElike dynamics within alkyl nanodomains. We discuss possible origins for the observed phenomenology. 


\section{INTRODUCTION}

The structural and dynamical properties of homopolymers with long alkyl-side groups, like the families of poly(n-alkyl methacrylates) (PnMAs), ${ }^{1-21}$ poly(n-alkyl acrylates) $(\operatorname{PnAAs})^{2,16,22}$ and poly(di-n-alkyl itaconates $)^{23-25}$ have been extensively studied during the last decades. Based on x-ray diffraction, it has been suggested that side groups of different monomeric units tend to aggregate forming self-assembled alkyl nanodomains [called polyethylene (PE)-like nanodomains]. ${ }^{12,16,24}$ From a dynamical point of view, these materials show a rich variety of relaxational processes, and the existence of two different glasstransitions was proposed. One was associated to the freezing of the motions within the alkyl nanodomains (PE-like, $\alpha_{P E}$ ) and the other with that of the main-chain dynamics $(\alpha) \cdot{ }^{9,12,16}$ Such a scenario mainly rests on calorimetric, dielectric and mechanical studies, that are not selective for the different processes at a molecular level, and on x-ray diffraction, where mostly carbons and oxygens are highlighted without the possibility of distinguishing mainchain and side-group contributions to the diffraction patterns. Therefore, employing a selective technique providing space/time resolution at a molecular level is essential to check the validity of this scenario. In a recent work $^{21}$ we showed that neutron scattering combined with isotopic labeling is the right tool to face this problem. The difference in scattering lengths of hydrogen and deuteron allows to separately study the different components or even molecular groups in soft materials. The neutron diffraction experiments of that work provided strong support to the nanosegregated structure in PnMAs. Moreover, selectively following the collective dynamics of alkyl nanodomains and that of the main-chain system we observed the emergence of anomalous behavior for the structural relaxation of the side-groups in high-order members. However, important questions like the existence of a 
glass-transition associated to this process are still unsolved.

In this work we present a thorough investigation of the structure and dynamics of two members of the family of PnMAs: poly(n-butyl methacrylate) PBMA (number of Cs in the alkyl side chain $\left.n_{C}=4\right)$ and poly(n-hexyl methacrylate) PHMA $\left(n_{C}=6\right)$. We have combined neutron diffraction, neutron spin echo, differential scanning calorimetry and dielectric spectroscopy (both in time and frequency domains) measurements. In particular, exploiting isotopic labeling, we have been able to follow by neutron scattering not only the collective dynamics at the main-chain and side-group levels, ${ }^{21}$ but also the self-motions of hydrogen atoms in the side-groups of PHMA. This has allowed us building comprehensive relaxation maps and putting the relaxation results into a perspective. Interestingly, the temperature dependence of the neutron diffraction results provides evidence for the occurrence of a glass-transition associated to the freezing of the inter-molecular correlations within the alkyl nanodomains. Furthermore, the comparison with bulk-PE data of the literature $26-28$ offers additional support to the nanophase hypothesis and the confined character of the side-group dynamics. We find a peculiar phenomenology for the dynamics involving sidegroup atoms (extremely stretched functional forms, Arrhenius-like behavior, decoupling of self- and collective motions). Different hypothesis are invoked to explain these observations: distributions of relaxation times along the side-groups and a more universal scenario based on a high-order Mode Coupling Theory $(\mathrm{MCT})^{29}$ transition, as it has been recently proposed for dynamically asymmetric polymer blends ${ }^{30}$ and proteins. ${ }^{31}$ 


\section{EXPERIMENTAL}

\section{A. Samples}

The samples investigated were fully deuterated PnMAs with 4 and 6 carbons in the alkyl side group: poly(n-butyl methacrylate) PBMA-d14 $\left(n_{C}=4\right)$ and poly(n-hexyl methacrylate) PHMA-d18 $\left(n_{C}=6\right)$. In the case of PHMA, a partially deuterated sample where the side group is protonated and the main chain (including the $\alpha$-methyl group) is deuterated (PHMA-d5), was also studied. As reference and when available, we will also show results obtained on deuterated poly(methyl methacrylate) PMMA-d8 $\left(n_{C}=1\right)$ and poly(ethyl methacrylate) PEMA-d10 $\left(n_{C}=2\right)$. All samples were supplied by Polymer Source. Their characteristics are shown in Table I. For the neutron scattering experiments, the samples were placed in flat aluminum sample holders. The thicknesses were such that transmissions of $\approx 90 \%$ were expected, allowing to neglect multiple scattering effects.

\section{B. Neutron Scattering Measurements}

The dynamical measurements were carried out using the neutron spin echo (NSE) technique which offers the highest energy resolution in neutron scattering. In addition, NSE is a unique technique since it determines the intermediate scattering functions directly in the time domain. This is achieved by coding the energy transfer in the scattering process for each neutron individually into its spin rotation. ${ }^{32}$ In this way, the application of precession magnetic fields before and after the scattering event results in a polarization of the neutron that depends only on the velocity difference of each neutron individually, irrespective of its initial velocity. These experiments finally access the normalized function 


$$
N S E(Q, t)=\frac{I_{c o h}(Q, t)-\frac{1}{3} I_{i n c}(Q, t)}{I_{c o h}(Q, 0)-\frac{1}{3} I_{i n c}(Q, 0)} .
$$

Coherent and incoherent terms are given by: ${ }^{33,34}$

$$
\begin{gathered}
I_{c o h}(Q, t)=\frac{1}{N}\left\langle\sum_{i, j=1}^{N} \bar{b}_{i} \bar{b}_{j} e^{i \vec{Q}\left[\vec{r}_{i}(0)-\vec{r}_{j}(t)\right]}\right\rangle \\
I_{i n c}(Q, t)=\frac{1}{N}\left\langle\sum_{i=1}^{N}\left(\overline{b_{i}^{2}}-\bar{b}_{i}^{2}\right) e^{i \vec{Q}\left[\vec{r}_{i}(0)-\vec{r}_{i}(t)\right]}\right\rangle
\end{gathered}
$$

with the momentum transfer $Q=4 \pi \sin (\theta / 2) / \lambda$, where $\theta$ is the scattering angle and $\lambda$ the wavelength of the incoming neutrons. $\bar{b}_{i}$ stands for the coherent scattering length of nucleus $i$ (see Table II) and $N$ is the number of atoms. $\vec{r}_{i}(t)$ is the position vector of atom $i$ at time $t$, and the brackets denote the thermal average. Coherent scattering arises from relative positions of atomic pairs at different times and, therefore, reflects collective dynamics. Incoherent scattering relates to the self-part of the vanHove correlation function (the probability for an atom to move by a distance $r$ in a given time $t$ ) revealing thus individual atomic motions.

On the other hand, neutron diffraction experiments assess the differential cross section, i. e., the number of scattered neutrons into a solid angle between $\Omega$ and $\Omega+d \Omega$, relative to the number of incident neutrons. ${ }^{33,34}$ As the intensity is integrated irrespectively of the energy transfer, these measurements reveal the static $(t=0)$ value of the coherent and incoherent intensities introduced above (Eqs. 2 and 3).

For the nuclei involved in our samples, Table II shows the values of the coherent and incoherent cross sections $\sigma_{c o h}^{i}=4 \pi \bar{b}_{i}^{2}$ and $\sigma_{i n c}^{i}=4 \pi\left(\overline{b_{i}^{2}}-{\overline{b_{i}}}^{2}\right)$. The values of the cross sections for the samples investigated $\sigma_{\operatorname{coh}(i n c)}=\Sigma_{i} \sigma_{\text {coh(inc) }}^{i}$, where the sum runs over all the nuclei in the monomer, can be found in Table I. From Tables I and II we can deduce that the intensity scattered by fully deuterated samples is predominantly coherent and reveals to 
a very good approximation the dynamic structure factor $S(Q, t)$, since the scattering lengths for all the atoms involved are very similar. In a neutron diffraction experiment, the static structure factor $S(Q)$ is thus obtained. Furthermore, fully protonated samples scatter most of the neutrons incoherently and energy-resolved measurements provide information on the self-motions of the hydrogens. The case of the partially deuterated samples is in between, and delivers additional independent information on other partial structure factors. At $Q$ values far from the partial structure factor peaks, the dynamic signal from these samples mainly reveals the incoherent scattering function of the protons in the sample.

\section{Structure: Neutron diffraction by D16}

The structure factors of PnMAs were investigated on the fully deuterated samples by means of the neutron diffractometer D16 at the Institut Laue-Langevin (ILL, Grenoble, France) with incoming wavelength $\lambda=4.54 \AA$. Thereby, the $Q$-range covered was between 0.2 and $1.9 \AA^{-1}$. The samples were investigated at different temperatures ranging from $100 \mathrm{~K}$ to $\approx T_{g}+70 \mathrm{~K}$. The correction for background scattering was done by properly subtracting the intensity scattered by the empty cell.

\section{Dynamics: Measurements on the NSE instrument IN11}

The NSE measurements were made at the IN11 instrument also at the ILL. A neutron wavelength of $5.5 \AA$ was used, covering Fourier times between $\approx 4$ ps and $\approx 8$ ns. Here we will consider results previously published in Ref. ${ }^{21}$ on the deuterated samples at two values of the momentum transfer: (i) at the common second maximum of the structure factor

for all samples $Q_{I I}=1.3 \AA^{-1}$ and (ii) at the corresponding position of the first maximum 
$\left(Q_{I}=0.5 \AA^{-1}\right.$ for PBMA-d14 and $Q_{I}=0.4 \AA^{-1}$ for PHMA-d18). More details can be found in that paper. We have also performed new measurements on the partially deuterated sample PHMA-d5 at $Q=1.5 \AA^{-1}$, accessing thereby the incoherent scattering function of the protons at the side-group. The temperatures investigated in this case were 175, 220, 265 and $310 \mathrm{~K}$. As NSE works in the time domain, the deconvolution of the measured spectra from resolution can be realized by simple division of the data collected at the temperature of interest by the instrumental resolution spectrum, that was determined from the sample at $2 \mathrm{~K}$. The weak background signal was carefully measured and properly subtracted from the experimental data. Due to the low intensity of the signal (reduced to $1 / 3$ due to the neutron spin flip in incoherent scattering) we employed measuring times of $18 \mathrm{~h}$ for each temperature.

\section{Dielectric and Calorimetric Measurements}

\section{Modulated Differential Scanning Calorimetry}

A differential scanning calorimeter TA Instrument Q2000 was used in "heat only" modulation mode to measure the calorimetric glass transition temperature of PnMAs. Modulated Differential Scanning Calorimetry (MDSC) allows to separate changes in the heat flow due to variations in the heat capacity $\left(C_{p}\right)$ of the sample from other type of heat contributions, that is, the so called reversible and non-reversible contributions. Samples of about $10 \mathrm{mg}$ were sealed in aluminum pans and heated at an average $5 \mathrm{~K} / \mathrm{min}$ rate with temperature modulation amplitude and period of $\pm 0.8 \mathrm{~K}$ and 60 s respectively. 


\section{Dielectric Measurements}

Time and frequency domain dielectric techniques were used to study the dynamics of the different PnMAs in a broad temperature and frequency range. In both cases, samples of typically $0.1 \mathrm{~mm}$ thickness in between two parallel gold plated electrodes of $20 \mathrm{~mm}$ diameter were used. Samples were dried in vacuum oven at $383 \mathrm{~K}$ for PEMA, $323 \mathrm{~K}$ for PBMA and $313 \mathrm{~K}$ for PHMA for 1 day prior DS and TSDC measurements. Thermally Stimulated Depolarization Currents (TSDC) technique in the time domain gives access to slow dielectric relaxation processes by measuring the current associated to the depolarization process during the controlled heating of a previously polarized specimen. The equipment consisted on a Keithley 6517A electrometer together with Novocontrol's Quatro temperature and WinTSC controllers. The initial polarization was obtained by applying a 200V voltage electric field at a temperature well above the calorimetric glass transition: $368 \mathrm{~K}$ for PEMA, $313 \mathrm{~K}$ for PBMA and $283 \mathrm{~K}$ for PHMA, and then cooling down the sample to $138 \mathrm{~K}$, where the polarization remains frozen after switching off the electric field. Depolarization current measurements were performed at a heating rate of $5 \mathrm{~K} / \mathrm{min}$, the same rate used for DSC experiments. In the more conventional frequency domain broadband dielectric spectroscopy (BDS), the complex dielectric function $\epsilon^{*}(\omega)=\epsilon^{\prime}(\omega)-i \epsilon^{\prime \prime}(\omega), \omega=2 \pi f$, is accessed by measuring the frequency dependent impedance of a capacitor formed by the sample and the gold electrodes. Measurements were carried out using a high-resolution dielectric analyzer (ALPHA Novocontrol) over a $10^{-2}$ to $10^{7} \mathrm{~Hz}$ frequency range. The temperature range explored was between $120 \mathrm{~K}$ and $420 \mathrm{~K}$ for PBMA and between $120 \mathrm{~K}$ and $395 \mathrm{~K}$ for PHMA. Data were collected every $5 \mathrm{~K}$ in the high-temperature range and every $10 \mathrm{~K}$ at low temperatures (i. e., below $290 \mathrm{~K}$ for PBMA and $210 \mathrm{~K}$ for PHMA). The temperature stability was better 
than $0.5 \mathrm{~K}$. In addition to isothermal measurements, temperature scans at $1 \mathrm{~K} / \mathrm{min}$ rate and at frequencies betwen 4 and $10^{6} \mathrm{~Hz}$ were also recorded. These measurements are a fast and sensitive way to obtain a global view of the different relaxations.

\section{RESULTS}

\section{A. DSC}

Figure 1 shows the temperature dependence of the reversible heat capacity and its derivative for the different polymers investigated. The calorimetric glass transition temperature $T_{g}^{D S C}$ are shown in Table III. They were determined from the position of the maximum of the derivative curve, i.e. the inflexion point of the reversible heat capacity curve. With increasing side-group size, the glass-transition shifts toward lower temperatures (internal plasticization effect); moreover, the peak in the derivative becomes broader and broader, mainly in the low-temperature side. Table III also displays the values of $T_{g}^{\text {mid }}$ obtained from the middle point of the heat capacity curve. Due to the asymmetry of the process, there is a difference between $T_{g}^{\text {mid }}$ and $T_{g}^{D S C}$ that increases with increasing side-group length.

\section{B. TSDC and DS}

Figure 2 shows the TSDC results for the different polymers investigated. The recorded current as a function of temperature reaches different maxima as the dipoles present in the polymer chains become mobile enough to randomize their orientation. A glass transition temperature was determined from the position of the main maxima of the depolarizing

current, which we will refer to as $T_{g}^{T S D C}$ (see Table III). The decrease of this temperature with increasing side-chain length reflects the plasticization effect of the side-groups in a 
similar way as that determined by DSC. In addition to the main peak, the TSDC results of PEMA show another well resolved peak at around $240 \mathrm{~K}$, which we attribute to the secondary process $\beta^{D S}$. This contribution seems to be approximately in the same location for the three polymers. Finally, a small peak can be observed for PHMA at very low temperatures $(150-175 \mathrm{~K})$, which corresponds to a process we will refer to as $\beta^{D S}$ in the following.

Analyzing now the isothermal BDS loss curves, in the region above $\approx T_{g}+25$ only a main peak is visible that splits into the $\alpha^{D S}$ and $\beta^{D S}$-processes at lower temperatures. A large number of works in the literature have been focused on the dielectric characterization of these main relaxational processes in PnMAs. ${ }^{2,7-12,18}$ Our results agree with the body of reported data and therefore we will not explain in detail the data analysis. This was performed in the so-called additive approach, simultaneously fitting the measured real and imaginary parts of the complex permittivity to the sum of a conductivity term and one or two Havriliak-Negami (HN) functions ${ }^{35}$. The HN-relaxation function is expressed as

$$
\Phi_{H N}^{\star}(\omega)=\frac{1}{\left[1+\left(i \omega \tau_{H N}\right)^{\alpha}\right]^{\gamma}}
$$

where the HN shape parameters $\alpha$ and $\gamma$ vary between 0 and 1 and $\tau_{H N}$ is the HN-time. The characteristic times defined as the inverse of the frequency corresponding to the maxima of the loss $\left(1 / \tau=2 \pi f_{\max }=\omega_{\max }\right)$ are displayed as empty symbols in Figs. 3 and 4 . They can be obtained from the characteristic times of the HN-parameters through:

$$
\tau_{H N} \omega_{\max }=\left[\frac{\sin \left[\frac{\alpha \pi}{2(\gamma+1)}\right]}{\sin \left[\frac{\alpha \gamma \pi}{2(\gamma+1)}\right]}\right]^{1 / \alpha}
$$

We will now focus in more detail in the low-temperature behavior. As can be seen in Fig. 5 for PHMA, the losses show a weak peak $\left(\gamma^{D S}\right)$ at very low temperatures (filled diamonds 
at $120 \mathrm{~K}$ ). Another process can be found in the intermediate range between the $\gamma^{D S}$ and the $\beta^{D S}$-processes. Though weak, there is clearly an extra-contribution to the imaginary parts in the high-frequency range at intermediate temperatures and data there can not be described with a single HN function (see e. g. Fig. 5). This process is also evident in the isochrone representation of the temperature scans (see insert of Fig. 5). The dielectric strength of this process $\left(\beta^{\prime D S}\right)$ is about 0.07 , the corresponding activation energy is $0.52 \mathrm{eV}$. The characteristic times associated to these two low- $T$ relaxations $\left(\gamma^{D S}, \beta^{D S}\right)$ are also displayed in Figs. 3 and 4. It is worth mentioning that the characteristic times of all the secondary relaxations $\left(\beta^{D S}, \beta^{D S}\right.$ and $\left.\gamma^{D S}\right)$ are practically indistinguishable for PBMA and PHMA, in agreement with results from the literature. ${ }^{1-3,9}$

\section{Diffraction: Microphase Separation}

Figures 6(a)-(b) show the D16 results on the fully deuterated PBMA and PHMA samples, revealing thus their structure factors, for the different temperatures investigated. Two main maxima can be distinguished in $S(Q)$ : a main common peak (peak II) centered at $Q_{I I} \approx 1.3 \AA^{-1}$ and another peak (peak I) located at smaller $Q$-values $\left(Q_{I}^{P B M A} \approx 0.5 \AA^{-1}\right.$ and $\left.Q_{I}^{P H M A} \approx 0.4 \AA^{-1}\right)$.

In a previous work, ${ }^{21}$ by exploiting neutron selectivity for labeled molecular groups and comparing the diffraction data with those corresponding to bulk PE, we concluded that peak I in $S(Q)$ reflects correlations between the structural subunits conforming the main-chain system, while peak II arises from correlations between atoms located in side-groups of different monomers in an environment of other alkyl side-chains. In this way, we demonstrated the existence of PE-like nanodomains in high order PnMAs, supporting the nanophase hypothesis. These statements might also be extensible to the case of PEMA, though with some 
caution, since not so well developed separation is expected in this polymer. A detailed analysis of PEMA's $S(Q)$ will be addressed in a separate publication, where also MD-simulations will be considered. ${ }^{36}$

The clear separation of peaks I and II in $Q$-space allows the selective study at a molecular level of the dynamics associated to each of the subsystems, i.e., their structural relaxations. This information is provided by the NSE experiments at the two maxima.

\section{NSE at Peak I: Structural Relaxation at the Main-Chain Level}

Figure 7 compares the normalized dynamic structure factor of PBMA and PHMA with those of PEMA and PMMA at the corresponding $Q_{I}$ and 3 different temperatures. With increasing side-chain length, we observe (i) a decrease in the amplitude of the function and (ii) that the decay takes place at shorter timescales. This last effect is due to the plasticization of the main-chains by the alkyl side-groups. Independently of the length of the side groups, the decay of the dynamic structure factor above $\approx 2$ ps can be described by a stretched exponential or Kohlrausch-Williams-Watts (KWW) function

$$
\frac{S(Q, t)}{S(Q, 0)}=D W F \exp \left[-\left(\frac{t}{\tau_{w}}\right)^{\beta}\right]
$$

with a value of 0.5 for the stretching parameter $\beta$. The Debye-Waller-factor (DWF) is the amplitude accounting for the fast decay of the correlations in the microscopic regime below $\approx 2 \mathrm{ps}$ and $\tau_{w}$ is the characteristic time. We will refer to the characteristic time of the dynamic structure factor at $Q_{I}$ as $\tau_{s}^{M C}$. The $n_{C}$-dependence of $\tau_{s}^{M C}$ for the same three temperatures is shown in Fig. 8(a). Figure 9 and the relaxation maps [Figs 3 and 4] display its temperature dependence. 


\section{E. NSE at Peak II: Structural Relaxation within the Nanodomains}

At peak II $\left(Q_{I I} \approx 1.3 \AA^{-1}\right)$, NSE follows the temporal evolution of the correlations between the structural units within the nanodomains, i. e., the side-groups (SG) structural relaxation. The results obtained at different temperatures are shown in Fig. 10. The expected KWW with $\beta \approx 0.5$ clearly fails in describing these data [see e. g. the dotted line in Fig. 10(b)]. Interestingly enough, the decay is nearly perfectly logarithmic (solid lines through the points):

$$
\frac{S(Q, t)}{S(Q, 0)}=A-B \ln \left(\frac{t}{\tau}\right)
$$

Logarithmic decays lack a characteristic timescale. In order to quantitatively compare different results we define the characteristic time as the time where the normalized correlation function decays a factor $1 / \mathrm{e}$ of its value at $2 \mathrm{ps}$. This definition takes into account the microscopic decay parametrized by the $D W F$ in the KWW equation (Eq. 6) and the results are directly comparable with KWW times. For the high temperatures investigated, the values of the characteristic times can be direclty read off from the NSE data, that decay to small values in the experimental window. However, when the temperature is decreased the such defined characteristic time lies out of the NSE window. Then, we have assumed the logarithmic-like functional form to persist toward long times. The such defined characteristic time for the dynamic structure factor at peak II, $\tau_{s}^{S G}$, is shown as function of $n_{C}$ in Fig. 8(b) and as function of $T$ in Fig. 9 and in the relaxation maps [Figs 3 and 4]. We note that in the case of PBMA at $300 \mathrm{~K}$, where the observed decay is rather weak [see Fig. 10(b)] the uncertainties involved prevent a reliable determination of the characteristic time. 


\section{F. NSE on PHMA-d5: Incoherent Scattering from Side-Group Hydrogens}

Thanks to the weak coherent intensity scattered by PHMA-d5 in the region of $Q \approx 1.5 \AA^{-1}$ [see Fig. 11(a)], NSE experiments were feasible, revealing the incoherent scattering function of side-group protons. The results obtained are shown in Fig. 11(b). The functions are extremely stretched. KWW descriptions as those shown in the figure require very low values of the stretching parameter $\beta$ ( 0.24 for $175 \mathrm{~K}$ and 0.1 for the higher temperatures) as compared with the typical value of $0.5 .{ }^{37}$ Moreover, for high $T$ unrealistic values are found for the amplitudes $(D W F>1)$. At $T \approx 220 \mathrm{~K}$, in the crossover region between a convex curvature at lower temperatures and a concave curvature at higher temperatures, a logarithmic-like decay can be again identified for this correlator.

At the highest temperature investigated for incoherent scattering, the self-correlation function decays to almost 0 within the NSE window; no evidence for any elastic contribution (elastic incoherent structure factor, EISF) is present. This result is expected since the sample is well above the calorimetric glass-transition temperature. The characteristic time $\tau_{\text {self }}^{S G}$ at this temperature can be rather accurately determined in a similar way as for coherent scattering $\left[S_{\text {inc }}\left(Q, \tau_{\text {self }}^{S G}\right)=S_{\text {inc }}^{S G}(Q, 2 \mathrm{ps}) / e\right]$. At $265 \mathrm{~K}$ (rather close to $T_{g}^{D S C}$ ) and lower temperatures a possible elastic contribution -associated to the localized character of motions in the glassy state- cannot be resolved by these measurements. If it indeed exists, the characteristic timescale should be defined relative to the decay of the quasielastic contribution (time where the quasielastic contribution decays to $1 / \mathrm{e}$ of its value at $2 \mathrm{ps}$ ). We do not know a priori how large the elastic contribution should be, and this leads to a degree of uncertainty in the determination of the characteristic time for this function. The data at $265 \mathrm{~K}$ indicate that the elastic level should not be larger than $\approx 0.2$. We thus may assume 
that the quasielastic contribution amounts to at least $(1$-EISF) $=0.8$ in the glassy state. We have considered that the value of the timescale should be between those deduced for (1EISF $)=1$ and for $(1$-EISF $)=0.8$. The such obtained values of $\tau_{\text {self }}^{S G}$ and the corresponding error bars have been plotted as diamonds in Fig. 4.

\section{DISCUSSION}

\section{A. Structural Relaxations: Main-Chain versus Side-Group}

A qualitative difference is found for the structural relaxations at main-chain and sidegroup levels for high-order PnMAs. At the main-chain level we observe standard $\alpha$ relaxations in all cases: typical KWW functions with $\beta=0.5$ provide excellent descriptions of all the data (Fig. 7). For $n_{C} \geq 2$ it has also been shown that the MC-results at different temperatures collapse into a single master curve when the timescale is scaled with the viscosity temperature dependence. ${ }^{21}$ The stretching and scaling properties are predicted by the MCT for the structural relaxation of glass-forming systems as observed at the first struc-

ture factor peak. ${ }^{38}$ On the contrary, the structural relaxation of the side groups in PBMA and PHMA exhibits extremely stretched (logarithmic-like) decays (Fig. 10). This kind of functional form is never observed for the slow decay of the correlation function in regular glass-forming polymers. We note that for PEMA, the structure factor at this $Q$-value follows the standard KWW description with $\beta=0.5 .^{21}$ Thus, a novel effect in the structural relaxation of these nanodomains emerges when the alkyl group contains at least about 4 hydrocarbons.

We now discuss in detail the influence of the side-group length on the structural relaxations of both subsystems as observed by NSE. As previously commented, the plasticizing 
effect of the side groups is manifested at a molecular level in an acceleration of the decay of the correlations between main chains when $n_{C}$ is increased (Fig. 7). The structural relaxation within the nanodomains becomes also faster with increasing $n_{C}$ and tends to approach that in bulk $\mathrm{PE}^{28}$ as can be directly observed in the comparison of Fig. 12. We note that in the temperature range where data are compared with $\mathrm{PE}$, the later is above the melting point and its dynamics is extremely fast. In fact, it does not show the typical two-step decay but a continuous decrease of the correlations around the picosecond region. ${ }^{28}$ This kind of behavior can't either be described by standard KWW functional forms. The structural relaxation time of $\mathrm{PE}$ will therefore be also defined on the basis of the decay to $1 / \mathrm{e}$ the value of the normalized structure factor at 2 ps.

At least for the highest temperatures, both structural times $\tau_{s}^{M C}$ and $\tau_{s}^{S G}$ approximatelly follow a power-law dependence on $n_{C}$,

$$
\tau_{s} \propto n_{C}^{-\gamma}
$$

[see Fig. 8]. For MC relaxation, we obtain $\gamma=2.8$ for $450 \mathrm{~K}$ and 2.3 for $480 \mathrm{~K}$; the data at $420 \mathrm{~K}$ would be compatible with $\gamma=4$. For $\tau_{s}^{S G}$, the values of the exponent $\gamma$ are larger: $5.9(420 \mathrm{~K}), 4.1(450 \mathrm{~K})$ and $3.4(480 \mathrm{~K})$. In Fig. 8(b) we have also indicated the values of the structural relaxation timescales of bulk PE as horizontal dotted lines. They match the extrapolations of the power-laws in the region around $n_{C}=9-10$. Thus, the side-chain dynamics of poly(n-decyl methacrylate) would be expected to be very similar to that of bulk-like PE.

The tendency of $\gamma$ to increase with decreasing $T$ implies that the $T$-dependence of the structural relaxation becomes weaker with increasing side-group length. Figure 9 shows the temperature dependence of both, $\tau_{s}^{S G}$ and $\tau_{s}^{M C}$, for PEMA, PBMA and PHMA. At 
high $T$ (actually, in the whole range investigated in most cases), this dependence can, at least effectively, be described by an Arrehnius-law. Interestingly, we observe that, for a given polymer, the obtained apparent activation energy is rather similar for $\tau_{s}^{S G}$ and $\tau_{s}^{M C}: E_{a}^{P E M A}\left(\tau_{s}^{M C}\right)=1.17 \mathrm{eV}, E_{a}^{P E M A}\left(\tau_{s}^{S G}\right)=1.25 \mathrm{eV}, E_{a}^{P B M A}\left(\tau_{s}^{M C}\right)=0.85 \mathrm{eV}$, $E_{a}^{P B M A}\left(\tau_{s}^{S G}\right)=0.81 \mathrm{eV}, E_{a}^{P H M A}\left(\tau_{s}^{M C}\right)=0.62 \mathrm{eV}, E_{a}^{P H M A}\left(\tau_{s}^{S G}\right)=0.58 \mathrm{eV}$. On the other hand, in all cases $\tau_{s}^{S G}$ is always faster than $\tau_{s}^{M C}$. In PEMA the side-chains are short and their motions are expected to be strongly coupled with those of the main chains. The difference between $\tau_{s}^{M C}$ and $\tau_{s}^{S G}$ in this case is not very large (about a factor 10). With increasing side chain length the dynamics within the nanodomains becomes increasingly faster than at the main-chain level -a dynamic asymmetry clearly develops between main-chain system and PE-like nanodomains for high-order PnMAs. This dynamic asymmetry $\Delta$ can be quantified by the ratio between the structural relaxation times of the two subsystems. Considering the power-law descriptions [Eq. 8], $\Delta \propto n_{C}^{-\left(\gamma^{M C}-\gamma^{S G}\right)}$. At the highest temperatures we can deduce that $\Delta \approx n_{C}^{1.2}$.

\section{B. Relaxation maps}

In Figs. 3 and 4 we compare the results obtained by the different techniques for PBMA and PHMA respectively. In Panels (a) the DSC and TSDC results are presented, and in Panels (b) the characteristic timescales deduced from NSE and DS. When results corresponding to diverse experimental approaches are brought together one has to keep in mind the different nature of the observables accessed by the different techniques. Calorimetry is sensitive to 'mass-weighted' dynamical changes, i.e., global features. For simple homopolymers is it usually found that $T_{g}^{D S C}$ determined at the usual cooling/heating rate $(10$ or $20 \mathrm{~K} / \mathrm{min}$ ) approximately coincides with the temperature at which the segmental relaxation as monitored 
with dynamical techniques reaches a characteristic time of about $100 \mathrm{~s}$. On the other hand, dielectric spectroscopy relies on dipole reorientation effects so that it preferentially senses the dynamic processes associated to dielectrically active segments. PnMAs have a strong dielectric dipole at the acrylate moiety, which is at the interface between the main-chain and the side-group subsystems. As a consequence, we expect the motion of this main dipole (and therefore the dynamics reflected in the dielectric measurements) to be neither fully slaved nor completely decoupled from either of the two mentioned subsystems, but to reflect both dynamics probably with $T$-dependent weights. The most straightforward interpretation is that of the NSE results, that have revealed the structural relaxations in both subsystems and the H-self motions in the side-groups.

For both polymers an excellent agreement is found between $\tau_{s}^{M C}(T)$ and the DS data for the merged $\alpha \beta^{D S}$-process. Thus, at high temperature the dynamics at the interface as followed by DS seems to be completely dominated by the main-chain relaxation. Due to the limited NSE window, the comparison cannot be extended to the glass-transition regime. For both polymers, the main TSDC peak occurs when the maximum in the dielectric loss attributed to the $\alpha^{D S}$-relaxation corresponds to a timescale of $100 \mathrm{~s}$. In this region, we note that the TSDC results are slightly shifted toward lower temperatures with respect to the DSC peak. In fact, the glass-transitions determined from both techniques differ by about $10 \mathrm{~K}$, see Table III). This is also the case for other members of the PnMAs series. ${ }^{14}$ The difference suggests that in this temperature range the dynamics at the interface is faster than the global relaxation (driven by the main-chain motions). As the temperature decreases and main-chain dynamics becomes slower, the motion of the dipole at the interface would not be fully decoupled from the side-group subsystem anymore, driving thus slightly faster dynamics than that of the segmental main-chain relaxation. 
Let us now consider the structural relaxation of the nanodomains, directly observed at a molecular level by NSE and characterized by $\tau_{s}^{S G}(T)$. As previously mentioned, in the high temperature range $\tau_{s}^{S G}(T)$ is always faster than $\tau_{s}^{M C}(T)$. Due to the limited NSE resolution, it is extremely difficult to extrapolate this timescale to lower temperatures and define a 'PElike' glass-transition without additional information. Calorimetric data are not conclusive at all, as the DSC derivative in both polymers does not show any clear maximum at lower temperatures that could be identified with such a glass-transition, but an extremely broad feature instead, in agreement with previous data in the literature. ${ }^{14}$ On the other hand, it would be tempting to think that the characteristic time of the dielectric process labeled as $\beta^{D S}$ could provide an extrapolation of the structural relaxation times for the side groups, in particular in the case of PHMA (see Fig. 4). The existence of this dielectric process has also been reported for poly(di-n-alkyl itaconates) ${ }^{25}$ poly(n-alkyl acrylates), ${ }^{16}$ and even for PnMAs. ${ }^{8,9,12,16,17}$ In the PnMAs series the intensity of the process is extremely low: Floudas et al. ${ }^{8}$ have reported a dielectric strength of 0.06 and an activation energy of $0.5 \mathrm{eV}$ for this process in a PDMA $\left(n_{C}=10\right)$ sample. These values are in very good agreement with our results in PHMA and PBMA. The authors have related it to 'a localized hindered rotation of the PE-like side chain' as it shows the same activation energy as reported for the mechanical $\gamma$-relaxation of PE. ${ }^{39}$ In those works, this relaxation was in fact identified with the structural relaxation of the PE-like nanodomains $\left(\alpha_{P E}\right)$. However, in our opinion the identification of a dielectric relaxation mechanism at the interface with the structural relaxation within the nanodomain is not straightforward. Therefore, we will look for a microscopic signature of the glass-transition within the nanodomains in the neutron scattering results. 


\section{Microscopic Signature of a Glass-Transition within the Nanodomains}

In Figure 6 we can see that peak II (revealing SG/SG correlations) shifts toward lower $Q$-values when heating the sample. This behavior is usually found in structure factor peaks reflecting intermolecular correlations and is attributed to thermal expansion. Figure 13 shows the $T$-dependence of the position of this peak $\left(Q_{I I}\right)$ for PBMA and PHMA. At high $T$, the results of both polymers are very similar. Interestingly enough, in the case of PHMA the $T$-dependence of $Q_{I I}$ there is just identical to that observed in bulk PE above the melting point (solid line through the points), where it can be followed. ${ }^{28}$ These observations support again the interpretation of this peak as of inter-molecular origin within the alkyl nanodomains. At low temperatures, the $T$-dependence of $Q_{I I}$ becomes much weaker, showing an abrupt decrease in the derivative in a temperature range that depends on the polymer $(150 \ldots 200 \mathrm{~K}$ for PHMA and $225 \ldots . .275 \mathrm{~K}$ for PBMA). In standard homopolymers such a transition takes place at the glass-transition temperature where the thermal expansion changes from that in the supercooled liquid state to that in the glassy state. We recall however that the calorimetric glass-transitions in these systems occur at much higher temperatures $\left(T_{g}^{D S C}(P H M A)=268 \mathrm{~K}, T_{g}^{D S C}(P B M A)=304 \mathrm{~K}\right)$. This behavior can thus be considered as a clear microscopic signature of the existence of a glass-transition (different from that at the main-chain level) within the nanodomains. Moreover, our data allow the direct determination of the temperature of this transition: $T_{g}^{S G}=175 \pm 25 \mathrm{~K}$ for PHMA and $T_{g}^{S G}=250 \pm 25 \mathrm{~K}$ for PBMA. While the value determined for PHMA is compatible with that proposed by Beiner et al. ${ }^{16}$ from DS measurements, in the case of PBMA we observe a substantially higher $T_{g}^{S G}$-value. From our diffraction data it would be extremely difficult to support that the actual SG-glass transition in PBMA could be located around $160 \mathrm{~K}$, as 
suggested by Beiner et al. ${ }^{16}$

We can now add this information to our relaxation maps (Figs. 3 and 4). The shadowed areas indicate the regions where we would expect the glass-transition of the side groups, i. e., $\tau_{s}^{S G}\left(T_{g}^{S G}\right)=1 \ldots 100 s$. For PBMA the SG-glass transition would take place at temperatures where the dynamical process of the interface labeled as $\beta^{D S}$ becomes extremely slow. This process has been investigated using multidimensional NMR and proposed to be due to a step-like side-chain rotation of the carboxyl group $\left(180^{\circ} \pm 20^{\circ}\right.$ flips $)$. Due to the asymmetry of the side group, flips need a main-chain rearrangement to occur. It was shown that it happens through rotations of about $20^{\circ}$ around the local chain axis. ${ }^{40}$ Thus, the complete relaxation of the side-group correlations seems to be arrested when such motions are strongly suppressed. Furthermore, we note that in this polymer the $T$-dependence of $\tau_{s}^{S G}(T)$ would not be very different from that shown by $\tau_{s}^{M C}(T)$. Thus, though faster, the dynamics of the side-groups in PBMA seems to be to some extent still coupled to that of the main chains. It is also clear that in this polymer the relaxation $\beta^{D S}$ cannot be identified with the structural relaxation of the nanodomains: in the region of temperatures compatible with $T_{g}^{S G}$, the characteristic time of the dielectric process is of about $10^{-5} \mathrm{~s}$. We thus support the interpretation of this process in PBMA as a secondary relaxation $\left(\beta_{P E}\right)^{20,41}$ rather than a dynamic glass-transition $\left(\alpha_{P E}\right) \cdot{ }^{16}$

In the case of PHMA, to compatibilize the values of $\tau_{s}^{S G}$ observed by NSE with the region $\tau_{s}^{S G}\left(T_{g}^{S G}\right)=1 \ldots 100 \mathrm{~s}$ (see Fig. 4), a rather weak temperature dependence (almost Arrheniuslike) toward low temperatures should be assumed for $\tau_{s}^{S G}(T)$. The collective relaxation of the side-groups in PHMA would be much faster not only than the main-chain structural relaxation but also than the $\beta^{D S}$-process: there is a possibility of fully relaxing the intermolecular correlations within the nanodomains without the aid of the motions involved 
in such processes. Finally, we note that the characteristic times of the $\beta^{D S}$-relaxation could in principle be compatible with the extrapolation of $\tau_{s}^{S G}$ toward low temperatures to match the location of $T_{g}^{S G}$. Thus, in this polymer apparently the dynamics revealed by the $\beta^{\prime D S}$-relaxation at the interface could show similar characteristic times to those of the actual SG-structural relaxation. However, it would be difficult to identify the dielectric process $\beta^{D S}$ with such dynamic glass-transition $\left(\alpha_{P E}\right)$ in PHMA. The main reason is that the $\beta^{D S}$ relaxations in PHMA and PBMA are nearly indistinguishable and we have shown above that for PBMA this process is different from the structural SG-relaxation. In this sense, this process could be interpreted as a kind of Johari-Goldstein relaxation of the alkyl nanodomains also in higher-order PnMAs, as suggested by Ngai and Beiner. ${ }^{41}$

\section{Self-Motions of PHMA Side-Group Hydrogens}

The decoupling observed between MC and SG motions in PHMA asks for a detailed investigation of the atomic motions in the side group of this polymer. At first sight, two features of the incoherent structure factor of SG-hydrogens in PHMA are salient: (i) the unusually stretched spectral shape, and (ii) the unexpectedly fast dynamics. To our knowledge, the results shown in Fig. 11 are the first experimental observation of a transition from convex to concave curvature and a logarithmic decay in the incoherent scattering function of a glass-forming homopolymer. The other surprising feature is that this dynamics, active in the nanosecond window, is observed at temperatures close and well below the calorimetric glass-transition of this sample (see Fig. 4). Thus, the side-group hydrogen motions show characteristic timescales of some nanoseconds even deep in the glassy state. Of course, fast methyl-group rotations at the side-group ends are expected to contribute to this signal. However, methyl hydrogens only represent $3 / 13$ of the protons in the side chain; more- 
over, their rotations do not fully relax the scattering function (at this $Q$-value, they lead to $\left.\mathrm{EISF}^{M G}=0.45\right)$. Therefore, the detected mobility is not attributable to only methyl group rotations, but has to reflect other fast motions within the side-group backbone.

Comparing self- and collective dynamics of side-groups, a third astonishing observation arises: an extreme difference in the characteristic time of the two kinds of dynamics. This can be realized in Fig. 14 for the closest conditions available. The collective relaxation observed at peak II and $330 \mathrm{~K}$ decays to $1 / \mathrm{e}$ at about $40 \mathrm{~ns}$, while the self correlation function of protons at $310 \mathrm{~K}$ and $Q=1.5 \AA^{-1}$ relaxes at $70 \mathrm{ps}$, a more than two orders of magnitude shorter time. As we show in the following, invoking deGennes narrowing $\tau_{c o h}=S(Q) \tau_{\text {self }}$ is clearly not enough to account for such a difference. To estimate $\tau_{\text {self }}\left(Q_{I I}=1.3 \AA^{-1}\right)$ starting from the observed value at $1.5 \AA^{-1}$ we use the typical $Q$ dependence of the incoherent times $\tau_{\text {self }} \propto Q^{-4} \cdot{ }^{37}$ Assuming $S\left(Q_{I I}\right) \approx 2$, the deGennes narrowing predicts $\tau_{c o h}(310 K) \approx 0.25 \mathrm{~ns}$. However, $20 \mathrm{~K}$ above, a much longer timescale (160 times) is observed for collective motion. The difference between the characteristic times for self- and structural times in PHMA can also be appreciated in the relaxation map (Fig. 4). In the $T$-range investigated, $\tau_{\text {self }}^{S G}$ shows an apparent activation energy of $0.27 \mathrm{eV}$, much weaker than that presented by the collective counterparts. On the other hand, the timescale deduced for the secondary $\gamma^{D S}$-relaxation is rather compatible with $\tau_{\text {self }}^{S G}$. Apparently, in this process the dipoles at the interface reflect the motions directly observed by NSE on the SG-atoms.

The decoupling of self- from collective motions can be interpreted as a signature of localized dynamics where the individual relaxation events are nearly decoupled from the decay of the inter-particle correlations. This localization suggests a confined character of the sidegroup dynamics. The dynamics under confinement for the alkyl groups in PnMAs was also 
discussed by Beiner et al. ${ }^{16}$

\section{E. Comparison with Bulk Polyethylene}

It is very instructive to compare PHMA-d5 results on the SG-hydrogen dynamics with those reported on bulk PE in a similar T-range (Fig. 15). We recall that there PE is semicrystalline. Quasilelastic neutron scattering investigations on hydrogenated semicrystalline PE were first reported by T. Peterlin-Neumaier and T. Springer ${ }^{26}$ in the temperature range $\approx 250 \ldots 335 \mathrm{~K}$. The quasielastic contribution to those spectra was attributed to the relaxation in the non-crystalline regions, and the data were interpreted in terms of protonic jumps between equidistant sites on the periphery of a circle of radius $2.5 \AA$. We note that the characteristic times deduced for such motions very nicely agree with those obtained in this work for PHMA SG-hydrogens in the same temperature range (see Fig. 15). Moreover, the value of the EISF corresponding to the geometry assumed for bulk PE (0.02) would be perfectly compatible with our results [see Fig. 11(b)]. In Ref. ${ }^{26}$, the process observed was identified with the so-called $\gamma$-process in PE as seen by NMR. In a recent work ${ }^{27}$ on semicrystalline PE combining different techniques (mechanical spectroscopy, positron anihilation, X-rays and NMR) it has been shown that in fact there exist two $\gamma$ mechanisms in PE. Our data perfectly match that with the highest activation energy $(0.25 \mathrm{eV})$, the so-called $\gamma_{2}$-process (see Fig. 15). The authors in Ref. ${ }^{27}$ interpret this relaxation mainly as due to the motion of the $\mathrm{C}-\mathrm{C}$ central bond of a short segment (e. g., three to four $\mathrm{CH}_{2}$ ) within interfacial-like amorphous phase. The coincidence of timescales of PHMA side-group hydrogens with the $\gamma_{2}$-process in semicrystalline PE would thus again support the hypothesis of PE-like nano-domains in this polymer. Furthermore, the similar dynamics observed in both cases seems to correspond to a confined situation (confinement by the nearby crystalline 
phases in pure PE, confinement by the rigid main-chain segments in PnMAs).

\section{F. Possible Scenarios}

The peculiarities observed for the dynamics of side-groups in the high-order PnMA members (in particular, in the most thoroughly investigated PHMA) can be summarized as:

(i) Extremely stretched (even logarithmic-like) decays for both, self and collective motions; convex to concave crossover for self-motions.

(ii) Possibly Arrhenius-like temperature dependences.

(iii) Very different characteristic times for self and collective motions.

(iv) Very fast characteristic times as compared with the main chain (i. e., development of dynamic asymmetry, that becomes more pronounced at low temperatures).

Feature (i) -anomalous stretching- could be attributed to the natural presence of a strong gradient of mobilities along the side groups. Those side-group atoms anchored to the main chain would move strongly coupled to the main-chain atoms and thereby they would be relatively slow. On the other hand, those side-group atoms further away from the main chain would have more freedom and move as fast as PE-like atoms. In the framework of this qualitative picture we could invoke a broad distribution of relaxation times ranging from rather short times - similar to those found in PE melts - to the relaxation times characteristic of main-chain dynamics in each case. Similar ideas have been used to interpret the broad dielectric relaxation function observed in some diblock-copolymers (see, e. g., ${ }^{42}$ ).

Deducing a distribution function of atomic mobilities / relaxation times is not straightforward, mainly in the case of coherent scattering. Here we may consider the simplest scenario for the incoherent results on PHMA. Let us label the $\mathrm{C}$ atoms along the side group with the index $i . \quad i=1$ corresponds to the aliphatic $\mathrm{C}$ linked to the oxygen atom, $i=2$ to the 
next $\mathrm{C}, \ldots i=6$ to the end (methyl) C. As the simplest approximation to a gradient of mobilities along the side-group, we assume that the characteristic times $\tau_{i}$ are equispaced in the $\operatorname{logarithmic}$ scale $\left(\Delta \log \tau=\log \tau_{i}-\log \tau_{i+1}\right.$ is constant) and the relaxation for each $\mathrm{H}$ is 'standard' (KWW with $\beta=0.5$ ). With this kind of simple approach a reasonable description of the experimental results can be achieved, as can be seen in Fig. 11(b). The corresponding characteristic times for H-mobilities would span over the vertical lines shown in Fig. 15 (see the values obtained for the parameters in Table IV). For one given $\mathrm{H}$ the time would be $4(310 \mathrm{~K}), 6(265 \mathrm{~K}), 11(220 \mathrm{~K})$ and 27 (175 K) fold shorter than that of the previous one along the chain. The longest times $(i=1)$ deduced would be rather compatible with those observed by DS (i.e., for the interface) in the $\beta^{\prime}$-process (see Fig. 15, Table IV). This finding would support the consistency of this scenario. However, we note that (i) the extreme broadening of the distribution function could be unrealistic ( 7 orders of magnitude for $175 \mathrm{~K}$ ) and (ii) the shortest times are faster than those one could consider as reasonable for the low-temperature extrapolation of the characteristic times of bulk PE, as might be appreciated in Fig. 15 (at $310 \mathrm{~K}, \log \left[\tau_{6}(s)\right]=-11.46$, estimated value for bulk PE at similar conditions $\left.\log \left[\tau^{P E}(s)\right] \approx-10.9\right)^{28}$. Finally, we note that logarithmic decays naturally emerge in the framework of a general kind of models based on a hierarchically constrained dynamics so that the relaxation of the system involves a sequential — rather than a parallel — series of correlated processes with an increasing characteristic time. ${ }^{43}$ Such kind of models could perhaps provide an explanation of the behavior found.

Extremely broad functional forms were also envisaged in results on diverse systems confined within well-defined geometries (e. g., salol, ${ }^{44}$ poly(dimethyl siloxane), ${ }^{45}$ poly(methyl phenyl siloxane $)^{45}$ and water ${ }^{46}$ in pores, and water in clays ${ }^{47}$ ). Systems confined in ill-defined geometries, or the fast component in systems where the dynamics of the other component(s) 
are much slower (dynamically asymmetric systems) might show this effect as well. For example, quasilogarithmic relaxation has been recently reported for the plasticizer Tri-m-cresyl Phosphate (TCP) in PMMA. ${ }^{48}$ Simulated asymmetric polymer blends of both kinds, fully atomistic and coarse-grained ${ }^{30,49}$ and a mixture of soft spheres with different mobilities ${ }^{50}$ are additional examples. For a binary mixture of mobile and static spheres, ${ }^{51}$ which can be seen as a simplified model of liquids in confining media with interconnected voids, this behavior has also been reported. These last cases rule out connectivity effects as an essential ingredient for such an observation. In a recent work, S.-H. Chen et al. have also reported logarithmic decays in a hydrated protein. ${ }^{31}$ Other strongly interacting systems of very different nature like e. g. granular materials ${ }^{52}$ or spin glasses ${ }^{53}$ also exhibit logarithmically decaying time evolution. On the other hand, Arrhenius-like dependences of the $\alpha$-relaxation timescale have been reported for confined systems, e. g. poly(methyl phenyl siloxane) in pores of $25 \ldots 50 \AA$ of diameter ${ }^{45}$ or also for the fast component in dynamically asymmetric polymer blends, e. g. poly(vinyl methyl ether) blended with polystyrene. ${ }^{42}$ Finally, a spectacular decoupling between self- and collective motions has been observed in molten sodium disilicate for the alkali ions, that move much faster than the Si-O network. ${ }^{54}$ Thus, the emergence of the peculiar phenomenology observed in PHMA seems to be independent of the particular microscopic details or nature of the system and more general theoretical scenarios might be invoked to account for it.

One possible origin for the exotic behavior shown by the side-group dynamics in highorder PnMAs could be the confined character of the system, or, more generally, of the existence of a strong dynamic asymmetry between the different components. In multicomponent miscible systems the dynamics of the fast component could be effectively restricted ('dynamically confined') by the slower ones. In the case of dynamically asymmetric polymer 
blends, the anomalous dynamic features were interpreted in the framework of the MCT, invoking high-order MCT-transition. ${ }^{29}$ Recently, this scenario has also been proposed to explain the logarithmic relaxation in proteins. ${ }^{31}$ This type of transitions emerges when different arrest mechanisms are present. They have been explicitly observed in short-range attractive colloids $^{55}$ and in a fluid of hard spheres confined in a disordered matrix of strictly static obstacles. ${ }^{56}$ For the case of the fast-component in polymer blends, the two mechanisms proposed were bulk-like caging (imposed by the neighboring fast component) and confinement (due to the motional restriction exerted by the slow matrix). For the side-group atoms in the alkyl nanodomains of high order PnMAs, these both mechanisms can also be envisaged. Side-group atoms should first escape from the cage imposed by the surrounding side-groups within the PE-like nanodomain, and complete relaxation could only occur when the more rigid main-chain would also relax. We speculate that a similar MCT scenario could also apply for the anomalous dynamic features observed in the PnMAs system.

\section{SUMMARY AND CONCLUSIONS}

Neutron scattering combined with isotopic substitution provides clear evidences for the nano-segregation of main-chains and alkyl side-groups in high-order PnMAs and the existence of alkyl nano-domains. It also offers the unique possibility of selectively following at a molecular level the structural relaxations of both subsystems -main chains and side-groups-, and the self-motions within these subsystems. Moreover, the microscopic information provided by the diffraction patterns has allowed to establish the existence of a glass-transition temperature within the nanodomains and the determination of its value. The examination of the relaxation maps points to an interpretation of the relaxation $\beta^{D S}$ as a secondary relaxation and not as the proposed dynamical glass-transition $\alpha_{P E}$. We note however that 
in the case of poly(di-n-alkyl itaconates) the identification of a similar dielectric process with a structural relaxation was justified, since Vogel-Fulcher behavior with relatively large activation entropy was indeed found for such process for $n_{C}=6 .^{25}$

Exploiting the NS capabilities, we have demonstrated the emergence of exotic dynamical behavior for the side-groups of PnMAs with $n_{C} \geq 4$ (at least, logarithmic-like structural relaxation). A complete dynamical decoupling, however, is only observed for PHMA. There, in addition, an extremely 'strong' temperature dependence of the structural relaxation time is observed. Self-motions are characterized by anomalous relaxation at much faster timescales than the collective motions. The analogies found between the self-motions of the side-group H-atoms and the $\gamma_{2}$-relaxation process in semicrystalline PE strengthen the picture of confined PE-like dynamics within the alkyl nano-domains.

The development of such an exotic phenomenology seems to be common for a large number of systems of different nature, including many dynamically asymmetric systems. This finding suggests the possibility of a general high-order MCT scenario for this kind of systems as it has been proposed for miscible polymer blends and hydrated proteins. On the other hand, we have shown that the extreme stretching found can be well accounted for -at least for the incoherent scattering function- by a broad distribution of mobilities along the side-group. We note that the presence of distributions could have an impact also in the $Q$-dependence of the incoherent scattering function. ${ }^{57}$ Unfortunately, present data are restricted to a single $Q$-value. Future experiments providing information on the variation of the incoherent scattering with momentum-transfer will be important to discern between different interpretations. Finally, how the single chain dynamics is affected by the presence of the side-groups is another key question and will be subject of future neutron scattering investigations in these systems. 


\section{ACKNOWLEDGEMENTS}

We thank the Institut Laue-Langevin (ILL) in Grenoble (France) for neutron scattering facilities and the ILL scientists P. Fouquet, M. Maccarini, B. Deme and P. P. Deen for experimental help at the ILL. We are also thankful to Prof. A. Alegría for fruitful discussions. This research project has been supported by the European Commission NoE SoftComp, Contract NMP3-CT-2004-502235, the 'Donostia International Physics Center'. A. A. and J. C. acknowledge support from the projects MAT2007-63681, IT-436-07 (GV) and the Spanish Ministerio de Educacion y Ciencia (Grant No. CSD2006-53).

\section{REFERENCES}

1 Heijboer, J. In Physics of Non-Crystalline Solids; Prins, J.A., Ed.; North-Holland: Amsterdam, $1965 ;$ p 231.

2 McCrum, N.G.; Read, B.E., Williams, G. Anelastic, Dielectric Effects in Polymeric Solids; Wiley: London, 1967.

3 Cowie, J.M.G. J. Macromol. Sci.-Phys. B 1980, 18, 569.

4 Meier, G.; Fytas, G., Dorfmüller, T. Macromolecules 1984, 17, 957.

${ }^{5}$ Giebel, L.; Meier, G.; Fytas, G.; Fischer, E. W. J. of Polym. Sci. B: Polym. Phys. 1992, 30, 1291.

${ }^{6}$ Garwe, F.; Schönhals, A.; Lockwenz, H.; Beiner, M.; Schröter, K.; Donth, E. Macromolecules $199629,247$.

7 Schröter, K.; Unger, R.; Reissig, S.; Garwe, F.; Kahle, S.; Beiner, M.; Donth, E. Macromolecules 
$1998,31,8966$.

8 Floudas, G.; Stepanek, P. Macromolecules 1998, 31, 6951.

9 Beiner, M.; Schröter, K.; Hempel, E.; Reissig, S.; Donth, E. Macromolecules 1999,32, 6278.

10 Dudognon, E.; Bernès, A.; Lacabanne, C. Macromolecules 2001, 34, 3988.

11 Dudognon, E.; Bernès, A.; Lacabanne, C. Macromolecules 2002, 35, 5927.

12 Beiner, M. Macromol. Rapid Commun. 2001,22, 869.

13 Beiner, M.; Kabisch, O.; Reichl, S.; Huth, H. J. Non-Cryst. Sol. 2002, 307, 658.

14 Hempel, E.; Beiner, M.; Huth, H.; Donth, E. Thermochimica Acta 2002, 391, 219.

15 Pascui, O.; Beiner, M.; Reichert, D. Macromolecules 2003, 36, 3992.

16 Beiner, M.; Huth H. Nature Mater. 2003, 2, 595.

17 Hiller, S.; Pascui, O.; Kabisch, O.; Reichert, D.; Beiner, M. New Journal of Physics 2004, 6, 1.

18 Menissez, C.; Sixou, B.; David, L.; Vigier G. J. Non-Cryst. Sol. 2005, 351, 595.

19 Wind, M.; Graf, R.; Renker, S.; Spiess, H. W. Macromol. Chem. Phys. 2005, 206, 142.

20 Beiner, M. CP832, Flow Dynamics, The Second International Conference on Flow Dynamics, edited by M. Tokuyama and S. Maruyama, American Institute of Physics, 2006; p. 134.

21 Arbe, A.; Genix, A.-C.; Colmenero, J.; Richter, D.; Fouquet, P. Soft Matter 2008, 4, 1792.

22 Gaborieau, M.; Graf, R.; Kahle, S.; Pakula, T.; Spiess H. W. Macromolecules 2007, 40, 6249.

${ }^{23}$ Cowie, J. M. G.; Haq, Z.; McEwen, I. J.; Velickovic, J. Polymer 1981, 22, 327.

24 Arrighi, V.; Triolo, A.; McEwen, I. J.; Holmes, P.; Triolo, R.; Amenitsch, H. Macromolecules 2000, 33, 4989.

25 Genix, A.-C.; Lauprêtre, F. Macromolecules 2005, 38, 2786.

26 Peterlin-Neumaier, T.; Springer, T. J. of Polym. Sci.: Polym. Phys. Ed. 1976, 14, 1351.

27 Matsuo, M.; Bin, Y.; Xu, C.; Ma, L.; Nakaoki, T.; Suzuki, T. Polymer 2003, 44, 4325. 
Arbe, A.; Colmenero, J. Phys. Rev. E. 2009, 80, 041805.

29 Götze W.; Sperl, M.; Phys. Rev. E 2002, 66, 011405.

30 Moreno A. J.; Colmenero, J. J. Chem. Phys 2006, 124, 184906.

31 Lagi, M.; Baglioni, P.; Chen, S.-H. Phys. Rev. Lett. 2009,103, 108102.

32 F. Mezei, Neutron Spin Echo, Lecture Notes in Physics (Springer-Verlag, Heidelberg, 1980), Vol. 28 .

33 Lovesey, S. W. Theory of Neutron Scattering from Condensed Matter; Clarendon Press: Oxford, 1984.

34 Squires, G. L. Introduction to the Theory of Thermal Neutron Scattering; Dover Publication Inc.: New York, 1996.

35 Havriliak, S.; Negami, S. Polymer 1967, 8, 161.

36 Genix, A.-C.; Arbe, A.; Colmenero, J.; Richter, D. et al, in preparation.

37 Richter, D.; Monkenbusch, M.; Arbe, A.; Colmenero, J. Neutron Spin Echo in Polymer Systems, edited by Advances in Polymer Science (Springer Verlag, Berlin Heidelberg New York, 2005) Vol. 174.

38 Götze, W.; Sjögren, L. Rep. Prog. Phys. 1992 ,55, 241.

39 Pineri, M.; Berticat, P.; Marchal, E. J. Polym. Sci., Polym. Phys. Ed. 1976, 14, 1325.

40 Schmidt-Rohr, K.; Kulik, A.S.; Beckham, H.W.; Ohlemacher, A.; Pawelzik, U.; Boeffel, C. Macromolecules 1994, 27, 4733.

41 Ngai, K.L.; Beiner, M. Macromolecules 2004, 37, 8123.

42 Lorthioir, C.; Alegría, A.; J. Colmenero, J. Phys. Rev. E 2003, 68, 031805.

43 Brey, J.J.; Prados, A. Phys. Rev. E, 2001, 63, 021108.

44 Zorn, R.; Hartmann, L.; Frick, B.; Richter, D.; Kremer, F. J. Non Cryst. Sol. 2002, 307, 547. 
45 Schönhals, A.; Goering, H.; Schick, Ch.; Frick B.; Zorn, R. J. Non Cryst. Sol. 2005, 351, 2668.

46 Gallo, P. Phys. Chem. Chem. Phys. 2000, 2, 1607.

47 Swenson, J.; Bergman, R.; Longeville, S. J. Chem. Phys. 2001, 115, 11299.

48 Bingemann, D.; Wirth, N.; Gmeiner J.; Rössler, E. A. Macromolecules 2007, 40, 5379.

49 Genix, A.-C.; Arbe, A.; Alvarez, F.; Colmenero, J.; Willner, L.; Richter, D. Phys. Rev. E 2005, 72, 031808.

50 Moreno, A. J.; Colmenero, J. Phys. Rev. E 2006, 74, 021409.

51 Krakoviack, V. Phys. Rev. Lett. 2005, 94, 065703.

52 Jaeger, H.M.; Liu, C.-H.; Nagel, S.R. Phys. Rev. Lett. 1989, 62, 40.

53 Nordblad, P.; Svedlindh, P.; Lundgren, L.; Sandlund, L. Phys. Rev. B 1986, 33, 645.

54 Horbach, J.; Kob, W.; Binder, K. Phys. Rev. Lett. 2002, 88, 125502.

55 Sciortino, F.; Tartaglia, P.; Zaccarelli, E. Phys. Rev. Lett. 2003, 91, 268301.

56 Krakoviack, V. Phys. Rev. E 2007, 75, 031503.

57 Arbe, A.; Colmenero, J.; Monkenbusch, M.; Richter, D. Phys. Rev. Lett. 1998, 81, 590. 
TABLE I: Molecular weight $M_{w}$, polydispersity, deuteration level, tacticity (syndiotactic diads) of the samples investigated, coherent and incoherent cross sections of the monomers.

\begin{tabular}{|c|c|c|c|c|c|c|}
\hline Polymer & $M_{w}(\mathrm{~g} / \mathrm{mol})$ & $M_{w} / M_{n}$ & deut. $(\%)$ & syndio $(\%)$ & $\sigma_{c o h}($ barns $/ \mathrm{mon})$ & $\sigma_{i n c}($ barns $/$ mon $)$ \\
\hline PEMA-d10 & 18000 & 1.06 & 91 & 87 & 97.68 & 20.50 \\
\hline PBMA-d14 & 16000 & 1.06 & 94 & 84 & 131.15 & 28.70 \\
\hline PHMA-d18 & 16000 & 1.07 & 87 & 83 & 164.62 & 36.90 \\
\hline PHMA-d5 & 90000 & 1.28 & 71 & 82 & 145.45 & 428.00 \\
\hline
\end{tabular}

TABLE II: Coherent scattering lengths, and coherent and incoherent cross sections for neutrons of the nuclei composing the samples.

\begin{tabular}{|c|c|c|c|}
\hline \multicolumn{2}{|c|}{ Nucleus $\bar{b}(\mathrm{fm})$} & $\sigma_{\text {coh }}($ barns/atom $)$ & $\sigma_{i n c}($ barns/atom \\
\hline $\mathrm{H}$ & -3.7406 & 1.7583 & 80.27 \\
\hline $\mathrm{D}$ & 6.671 & 5.592 & 2.05 \\
\hline $\mathrm{C}$ & 6.6511 & 5.559 & 0 \\
\hline $\mathrm{O}$ & 5.803 & 4.232 & 0 \\
\hline
\end{tabular}

TABLE III: Glass-transitions obtained through different methods or definitions in the samples.

\begin{tabular}{|c|c|c|c|}
\hline Polymer & $T_{g}^{T S D C}(\mathrm{~K})$ & $T_{g}^{D S C}(\mathrm{~K})$ & $T_{g}^{m i d}(\mathrm{~K})$ \\
\hline PMMA & & 398 & 400 \\
\hline PEMA-d10 & 355 & 353 & 350 \\
\hline PBMA-d14 & 293 & 304 & 299 \\
\hline PHMA-d18 & 257 & 268 & 255 \\
\hline
\end{tabular}


TABLE IV: Parameters involved in the description of the incoherent data of PHMA-d5 in terms of simple distributions of relaxation times, compared with the characteristic time of the $\beta^{\prime D S}$ process in PHMA.

\begin{tabular}{llll}
$T(\mathrm{~K})$ & $\log \left[\tau_{1}(s)\right] \Delta \log \tau$ & $\left.\log \left[\tau(\beta)^{\prime D S}\right](s)\right]$ \\
\hline 175 & -2.41 & 1.43 & -0.4 \\
220 & -5.25 & 1.05 & -3.5 \\
265 & -6.69 & 0.81 & -5.5 \\
310 & -8.41 & 0.62 & -6.9
\end{tabular}




\section{FIGURE CAPTIONS}

Figure 1: Temperature evolution of the reversible heat capacity and its derivative for PMMA (a), PEMA (b), PBMA (c) and PHMA (d).

Figure 2: TSDC results on PEMA (circles), PBMA (squares) and PHMA-d5 (diamonds).

Figure 3: (a) DSC (lines) and TSDC (circles) results on PBMA. (b) Relaxation map of PBMA. The NSE timescales observed at peaks I (full circles) and II (full squares) are compared with results from dielectric spectroscopy (empty symbols, crosses; the dashed line shows the extrapolation of the characteristic times obtained for the $\gamma^{D S}$-relaxation). The shadowed area represents the region where the timescale of the structural relaxation of the alkyl nanodomains should be at the glass-transition, according to the diffraction results.

Figure 4: (a) DSC (lines) and TSDC (circles) results on PHMA. (b) Relaxation map of PHMA. The NSE timescales observed at peaks I (full circles), II (full squares) and for incoherent scattering (full diamonds) are compared with results from dielectric spectroscopy (empty symbols, crosses, pluses). The shadowed area represents the region where the timescale of the structural relaxation of the alkyl nanodomains should be at the glasstransition, according to the diffraction results.

Figure 5: Real (circles) and imaginary (squares) parts of the dielectric permittivity measured on PHMA at $255 \mathrm{~K}$. The losses at $120 \mathrm{~K}$ are also shown (diamonds). Insert: imaginary part of the dielectric permittivity at $\log [f(H z)]=1$ measured during a temperature scan at $1 \mathrm{~K} / \mathrm{min}$.

Figure 6: Differential scattering cross sections measured by means of D16 on PHMA-d18 (a) and PBMA-d14 (b) at the temperatures indicated.

Figure 7: Comparison of the normalized dynamic structure factor at peak I for polymers with different side-group length for $420 \mathrm{~K}$ (a), $450 \mathrm{~K}$ (b) and $480 \mathrm{~K}$ (c). Lines are fits to 
KWW functions with $\beta=0.5$.

Figure 8: Chain-length dependence of the characteristic times observed for the decay of the main-chain correlations (a) and for the correlations involving side-groups (b) at three temperatures. Solid lines are guides for the eye. The dotted lines in (b) indicate the corresponding timescales in pure PE.

Figure 9: Arrhenius plot of the structural relaxation times corresponding to main-chain (empty symbols) and side-group (full symbols) subsystems. Circles: PEMA; Squares: PBMA; Diamonds: PHMA. Lines are Arrhenius fits.

Figure 10: Normalized dynamic structure factor measured by NSE at peak II $\left(Q_{I I}=1.3 \AA^{-1}\right)$ for PHMA-d18 (a) and PBMA-d14 (b)). Solid lines are fits to logarithmic expressions [Eq. 7], and the dotted line shows a KWW function with $\beta=0.5$ for comparison.

Figure 11: Results on PHMA-d5 (deuterated main chain and protonated side-groups) at the different temperatures indicated: (a) Ratio between the coherent and incoherent differential cross sections measured by D7 and (b) NSE signal measured at $Q=1.5 \AA^{-1}$. Solid lines are fits to KWW equations. Dashed lines are descriptions in terms of distributions of 6 stretched exponentials with characteristic times equidistant in logarithmic scale (see text).

Figure 12: Comparison of the normalized dynamic structure factor at peak II for the three PnMAs (full circles: PEMA-d10; squares: PBMA-d14; diamonds: PHMA-d18) and pure $\mathrm{PE}^{28}$ (empty circles) for $420 \mathrm{~K}$ (a), $450 \mathrm{~K}$ (b) and $480 \mathrm{~K}$ (c). Solid lines are fits to logarithmic expansions [Eq. 7], and dashed lines KWW functions with $\beta=0.5$. The vertical line shows $t=2 \mathrm{ps}$ (fast dynamics limit). The procedure to define the characteristic timescale for the logarithmic-like decays is exemplified in (b). 
Figure 13: Temperature dependence of the position of the maxima corresponding to peak II for PBMA and PHMA. Lines show the $T$-dependences, where the solid line through high- $T$ data of PHMA has the slope reported for pure PE. ${ }^{28}$

Figure 14: Comparison between the decay of the dynamic structure factor at peak II (squares) and of the incoherent scattering function at $Q=1.5 \AA^{-1}$ (circles) and similar temperatures (330 K for the dynamic structure factor, $310 \mathrm{~K}$ for incoherent scattering function). Lines are fits to KWW equation with $\beta=0.1$ (self) and logarithmic function (collective). The horizontal dotted line shows the $1 / e$-value as reference.

Figure 15: Comparison between the characteristic times (squares: structural relaxation, diamonds: self-motions of hydrogens at $Q=1.5 \AA^{-1}$ ) obtained for the side-group motions in PHMA (full symbols) and in bulk PE above the melting point ${ }^{28}$ (empty symbols). The lines are PE results in the semicrystalline state: the solid line shows the times reported in Ref. ${ }^{26}$ for the jump-like motions and the dotted line that corresponding to the $\gamma_{2}$-process detected by NMR in Ref. ${ }^{27}$. Vertical dashed-dotted lines through $\tau_{\text {self }}^{S G}$ show the distribution of mobilities needed to describe the stretching of the self-motions in side-group hydrogens (Table IV). Crosses are the characteristic times of the dielectric $\beta$ '-relaxation in PHMA fitted by an Arrhenius law (dashed line) with an activation energy of $0.52 \mathrm{eV}$. 


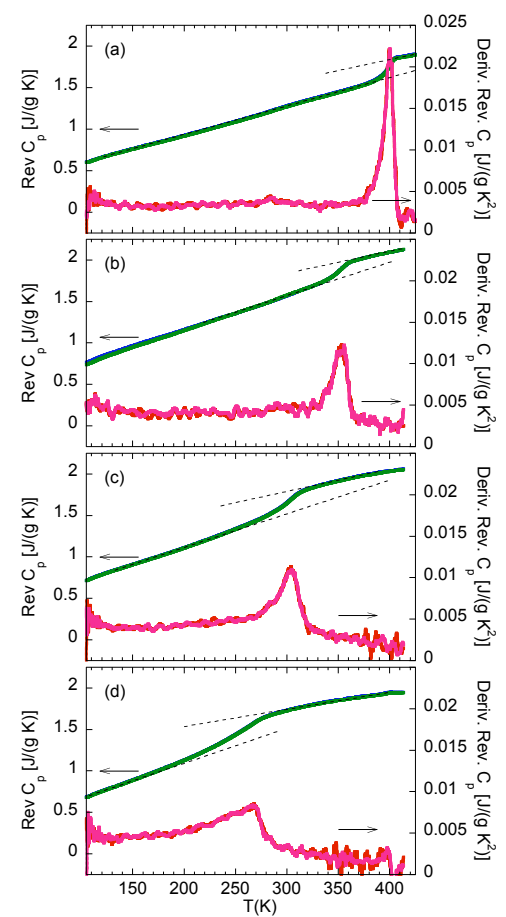

FIG. 1: 


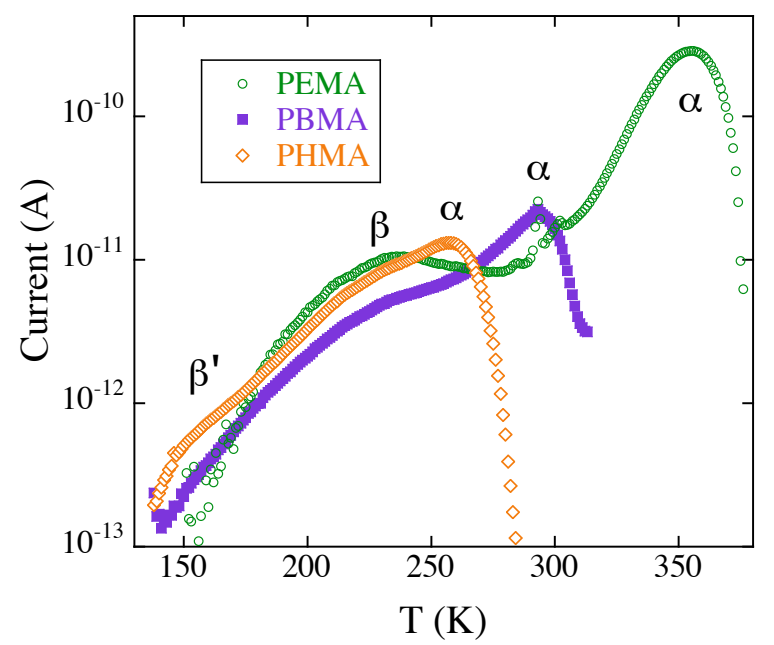

FIG. 2: 


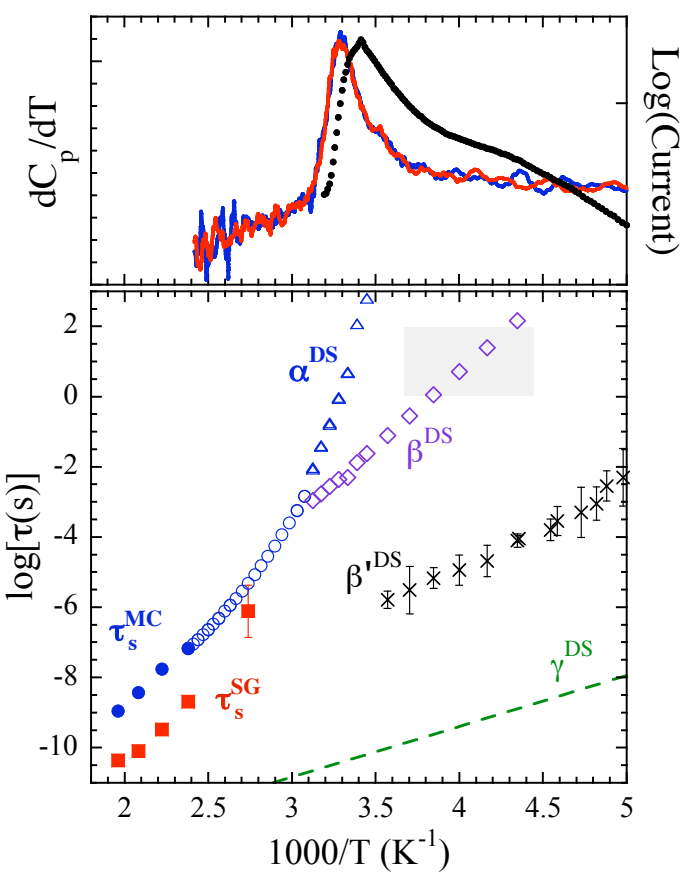

FIG. 3: 


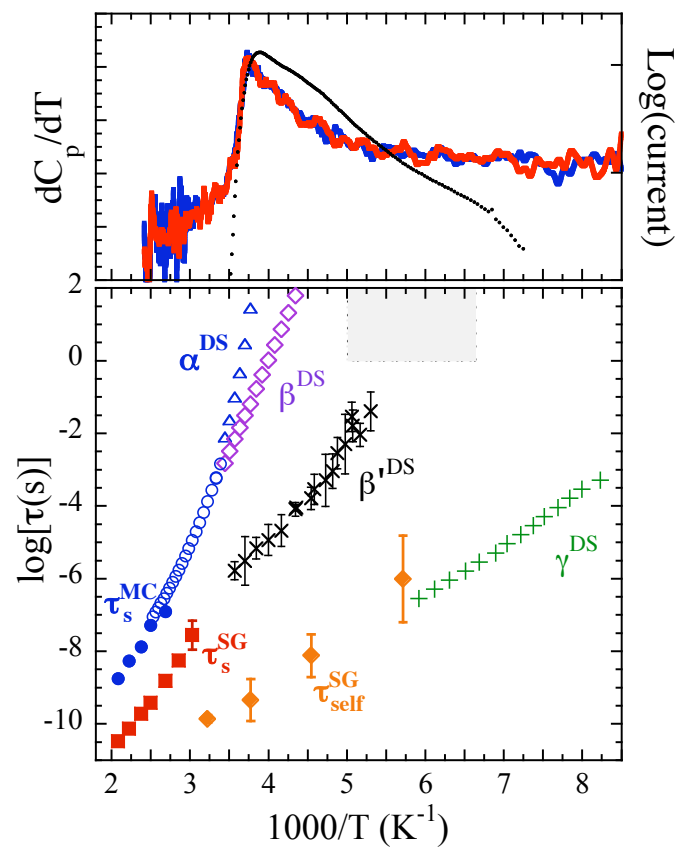

FIG. 4: 


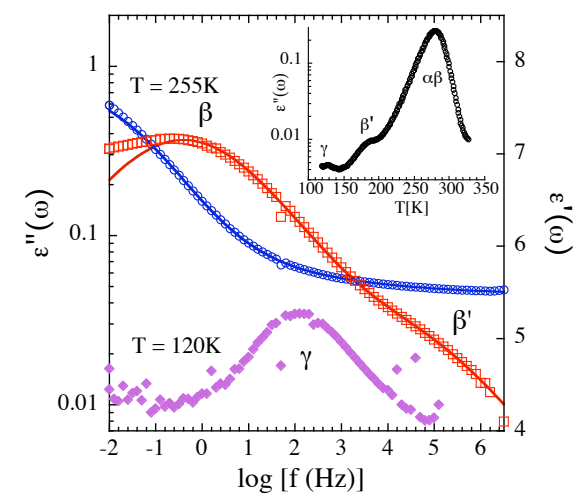

FIG. 5: 


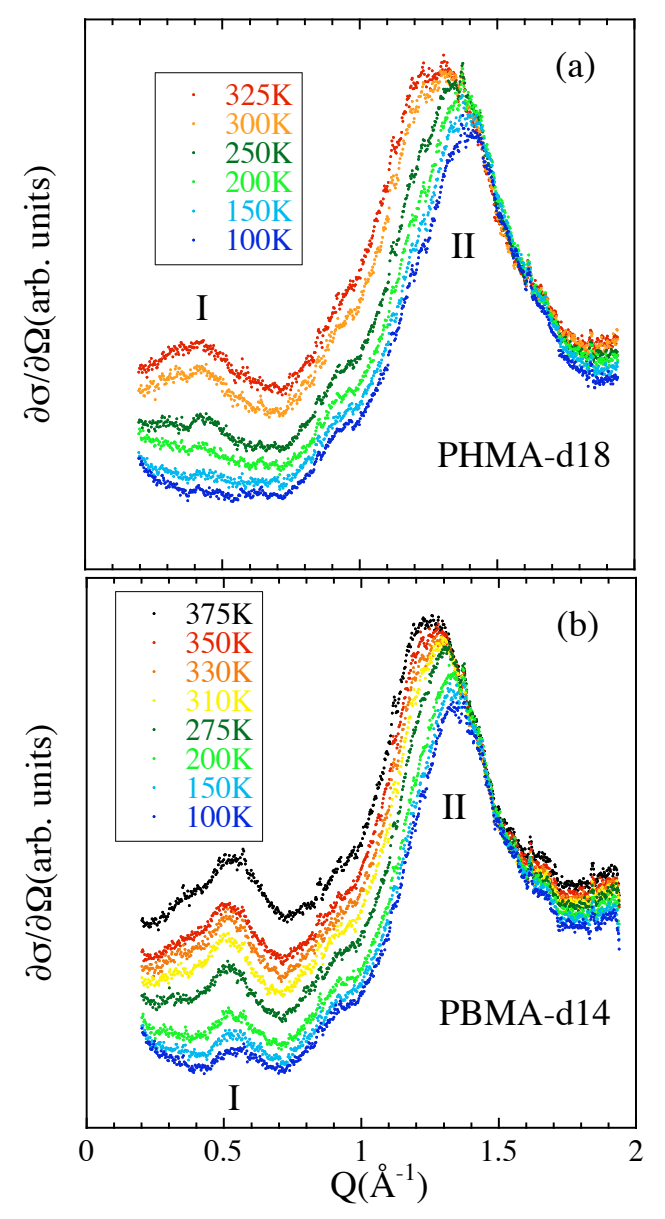

FIG. 6: 


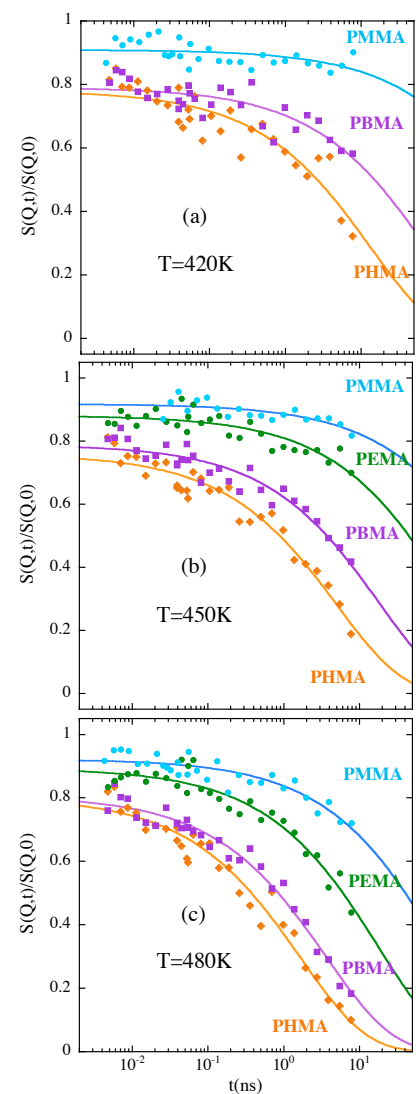

FIG. 7: 


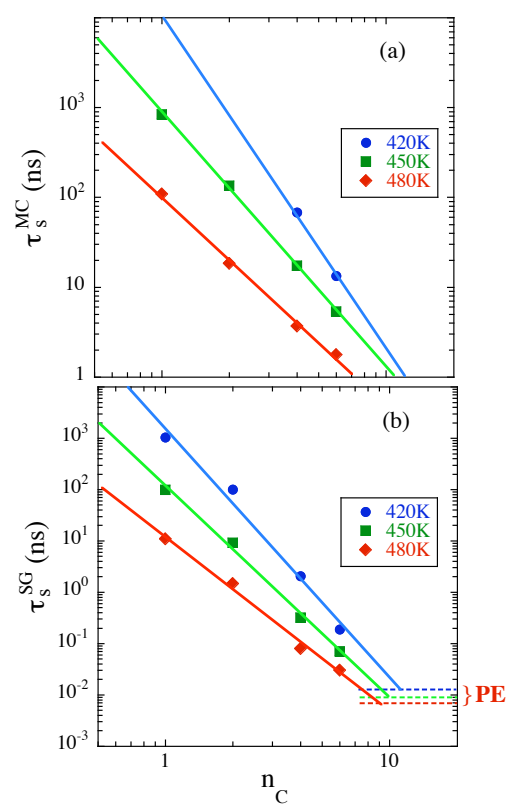

FIG. 8: 


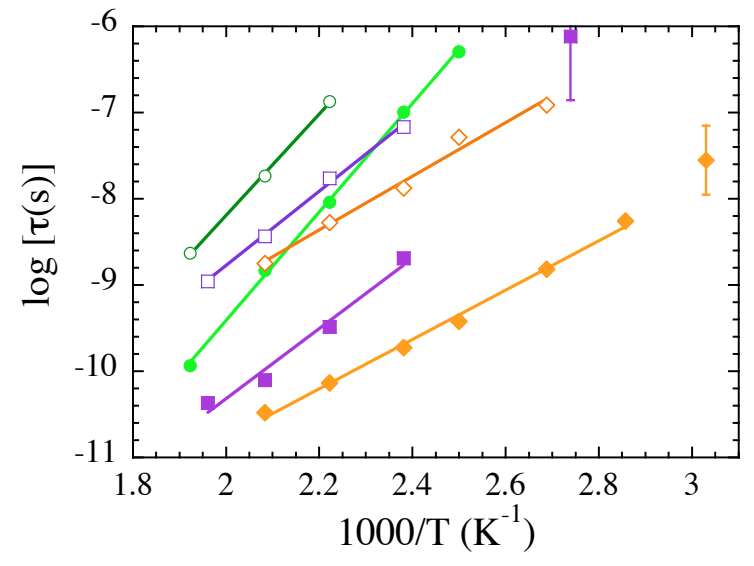

FIG. 9: 


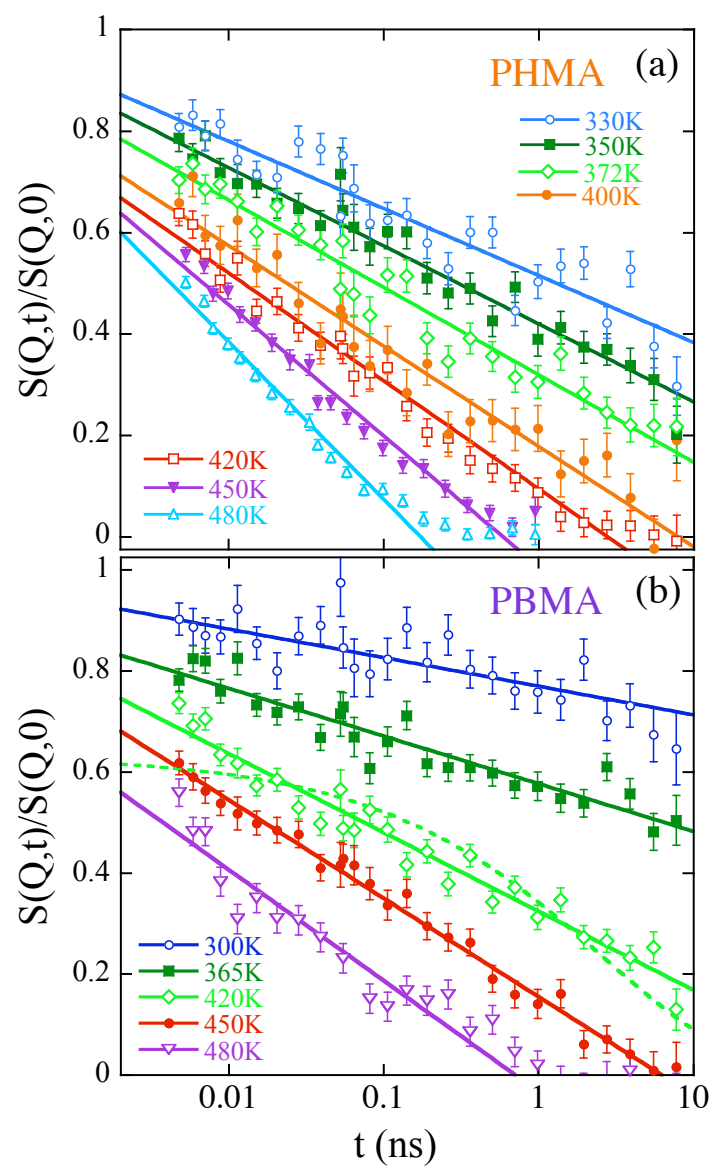

FIG. 10: 

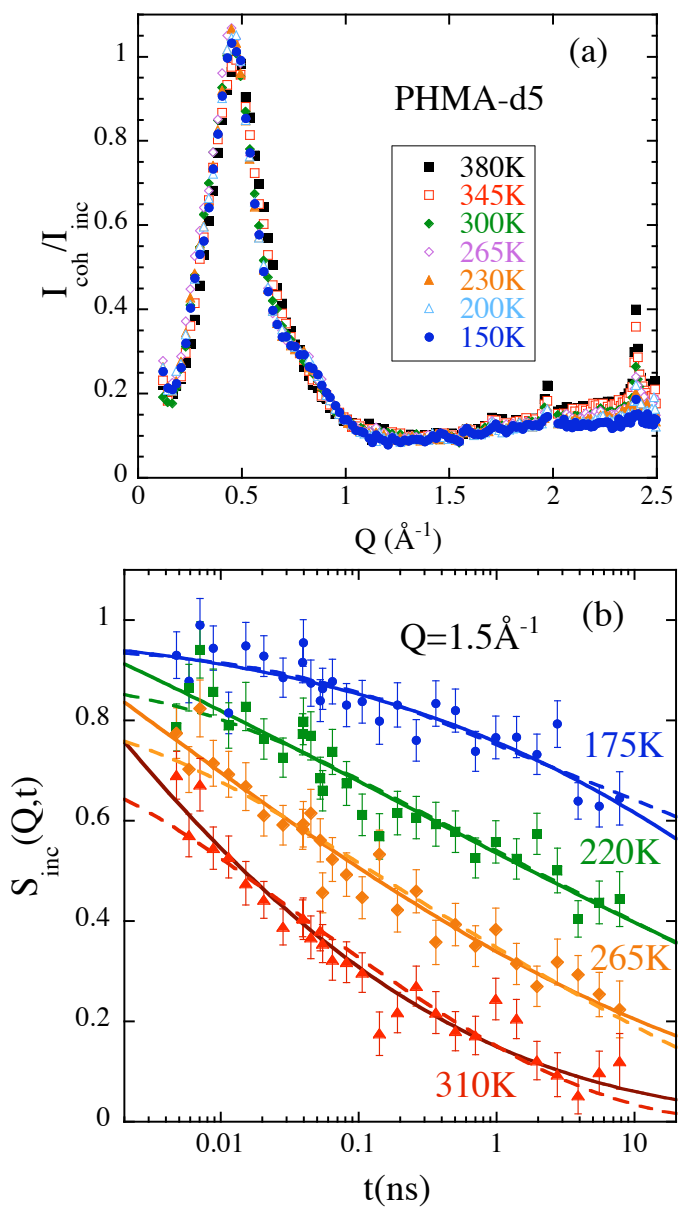

FIG. 11: 


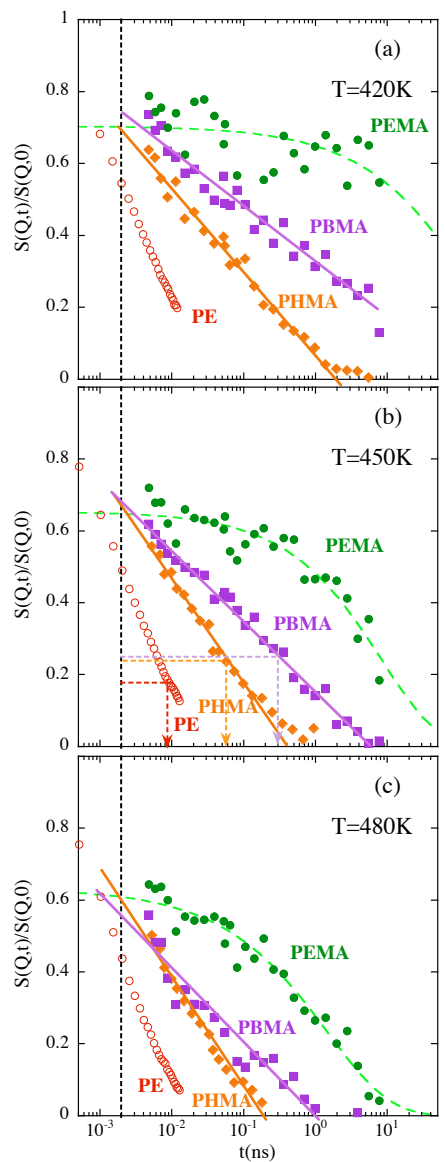

FIG. 12: 


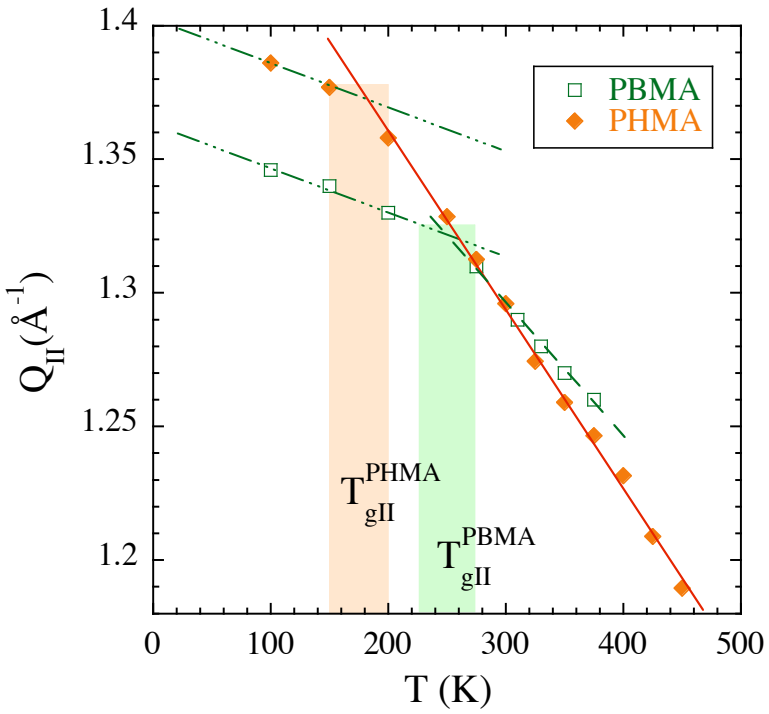

FIG. 13: 


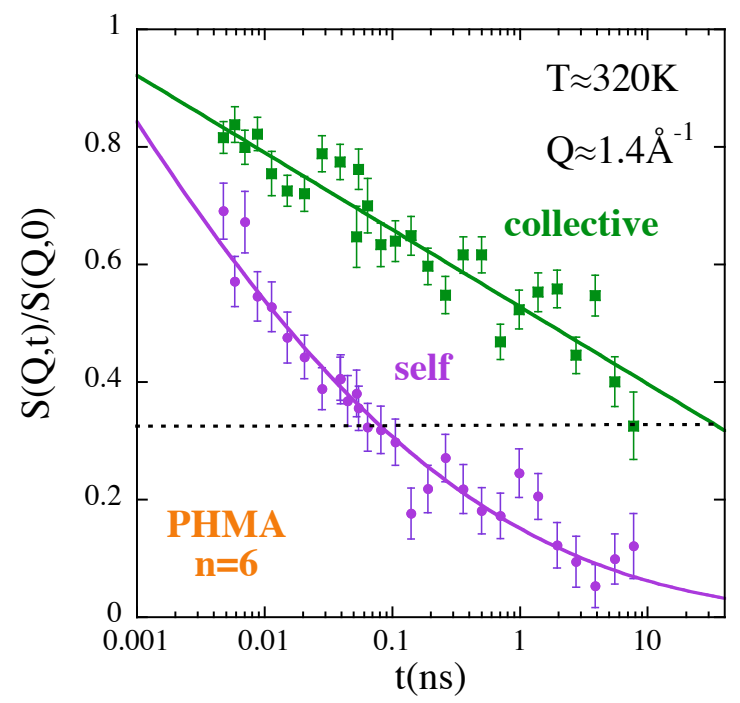

FIG. 14: 


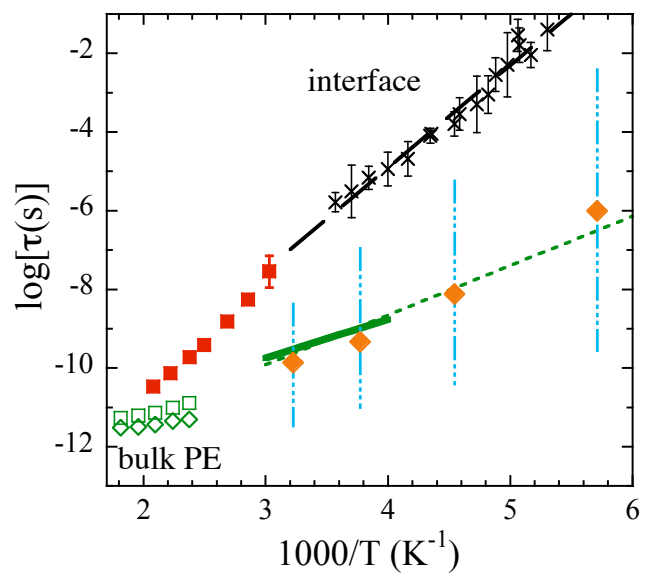

FIG. 15: 


\section{FOR TABLE OF CONTENTS USE ONLY}

Dynamics in Poly(n-alkyl methacrylates): A neutron scattering, calorimetric and dielectric study A. Arbe, A.-C. Genix, S. Arrese-Igor, J. Colmenero, D. Richter

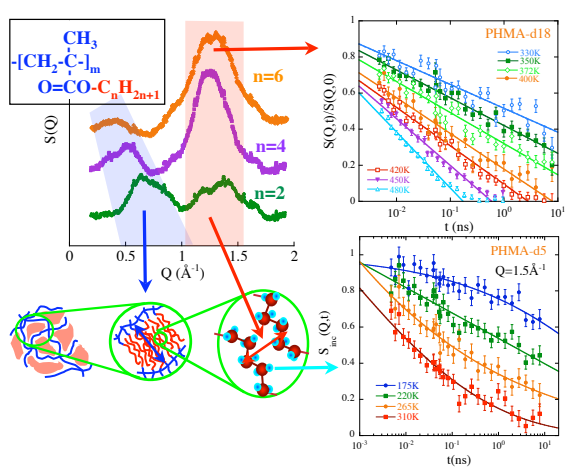

FIG. 16: 